\title{
Article
}

\section{Comparison of cryotherapy modality application over the anterior thigh across rugby union positions; a crossover randomized controlled trial}

Alexander, Jill, Rhodes, David, Birdsall, Daniel and Selfe, James Available at https://clok.uclan.ac.uk/33084/

Alexander, Jill orcid iconORCID: 0000-0002-6492-1621, Rhodes, David orcid iconORCID: 0000-0002-4224-1959, Birdsall, Daniel and Selfe, James (2020) Comparison of cryotherapy modality application over the anterior thigh across rugby union positions; a crossover randomized controlled trial. International Journal of Sports Physical Therapy, 15 (2). pp. 210-220.

It is advisable to refer to the publisher's version if you intend to cite from the work. $10.26603 /$ ijspt20200210

For more information about UCLan's research in this area go to http://www.uclan.ac.uk/researchgroups/ and search for < name of research Group>.

For information about Research generally at UCLan please go to http://www.uclan.ac.uk/research/

All outputs in CLoK are protected by Intellectual Property Rights law, including Copyright law. Copyright, IPR and Moral Rights for the works on this site are retained by the individual authors and/or other copyright owners. Terms and conditions for use of this material are defined in the policies page.

\section{CLoK}

Central Lancashire online Knowledge www.clok.uclan.ac.uk

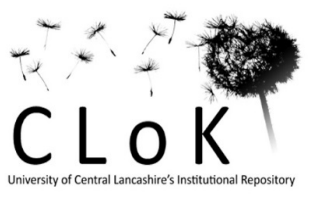




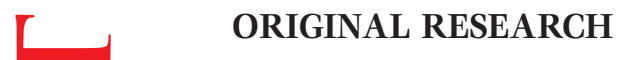 \\ COMPARISON OF CRYOTHERAPY MODALITY APPLICATION OVER THE ANTERIOR THIGH ACROSS RUGBY UNION POSITIONS; A CROSSOVER RANDOMIZED CONTROLLED TRIAL.
}

\author{
Jill Alexander MSc ${ }^{1}$ \\ Dr David Rhodes PhD $^{2}$ \\ Daniel Birdsall BSc ${ }^{1}$ \\ Prof. James Selfe DSc ${ }^{3}$
}

\begin{abstract}
Background: In deliberation of the diverse physical traits of rugby union and the known interference adipose tissue has on the ability to cool deeper tissues, evidence is required to understand the effect of cryotherapy modalities to provide optimum outcomes post-injury.
\end{abstract}

Purpose: To investigate differences in the cooling ability of three different cryotherapy modalities in a rugby union population in an attempt to describe optimum cooling protocols for the anterior thigh.

Study Design: Within-subjects randomized control crossover.

Methods: Twenty-one healthy male rugby union players took part. Skin surface temperature measured via thermal imaging camera (ThermoVision A40M, Flir Systems, Danderyd, Sweden) alongside Thermal Comfort and Sensation questionnaires following interventions of either Wetted Ice (WI), Crushed Ice (CI) applied in a polythene bag secured by plastic wrap, or CryoCuff® (CC), applied for 20-minutes over the anterior thigh. Participants were grouped by their typical playing position for the sport of rugby union; i.e. forwards and backs.

Results: Significant differences $(p=<0.05)$ in $\mathrm{T}_{s k}$ for all modalities compared to baseline and comparing post $\mathrm{T}_{s k}$ between CI and CC $(p=0.01)$ and WI to CC $(p=0.01)$ were displayed. Significantly greater reductions in $\mathrm{T}_{s k}$ noted immediately-post in the 'forwards' group $(p=\leq 0.05)$ compared to the backs' group for, all modalities $(p=\leq 0.05)$. Thermal Comfort and Sensation scores demonstrated significant changes baseline compared to post for all modalities $(p=<0.05)$. No significant differences were found when comparing between modalities for Thermal Comfort $(p=0.755)$ or Sensation $(p=0.225)$ for whole group or between positional groups.

Conclusions: Physiological responses to cooling differed across modalities with WI producing the greatest decrease in $\mathrm{T}_{\text {sk. }}$ Significant variability in $\mathrm{T}_{\text {sk }}$ was also displayed between positional factions. Results uphold the importance of the individualization of local cooling protocols when considering physical traits and characteristics within a rugby union population. Findings provide further understanding of the physiological responses to cooling through $\mathrm{T}_{\text {sk }} q u a n-$ tification in specific populations, helping to guide sports medicine practitioners on optimal cooling application development in sport.

Level of Evidence: Level $2 b$

Keywords: Cryotherapy, Rugby Union, Thermal Imaging, Movement System

${ }^{1}$ University of Central Lancashire, School of Sport and Health Sciences, Preston, Lancashire, UK.

${ }^{2}$ Institute of Coaching and Performance (ICaP), University of Central Lancashire, School of Sport and Health Sciences, Preston, Lancashire, UK.

${ }^{3}$ Manchester Metropolitan University, Faculty of Health, Psychology and Social Care, Manchester, UK.

The authors report no conflicts of interest.

\section{CORRESPONDING AUTHOR}

Jill Alexander, MSc

University of Central Lancashire

School of Sport and Health Sciences

Preston, Lancashire, PR1 2HE

United Kingdom

E-mail: JAlexander3@uclan.ac.uk 


\section{INTRODUCTION}

Selection and application of cryotherapeutic modalities in sport vary, with optimum exposure protocols still under debate in practice when considering clinical effectiveness and thermodynamics. ${ }^{1,2,3,4}$ Common cooling modalities include ice variations such as crushed, flaked, cubed, and wetted (typically crushed ice mixed with water); ice packs, gel packs, cold sprays, frozen peas, cryotherapy cuffs (CryoCuff ${ }^{\circledR}$ ) and coldwater immersion. ${ }^{2,5,6,7}$ Modalities are often applied via several methods for example with or without compression adjuncts. No definitive consensus is available on individual parameters of optimal cooling applications, either clinically or applicable to pitch side injury. Understanding the implications of different local cooling exposures are imperative to the management of sports injuries, ${ }^{5,8}$ with inadequate treatment paradigms being potentially detrimental to recovery timelines. ${ }^{4}$ Key cellular and physiological changes following cryotherapy are well reported through the of heat extraction from the body to achieve therapeutic effects. ${ }^{1,9,10,11}$ This includes an analgesic response and reductions in nerve conduction velocity, ${ }^{10}$ metabolism and inflammation. ${ }^{9}$ The efficacy of cryotherapy is often investigated through quantification of skin surface temperature $\left(\mathrm{T}_{s k}\right)$, via thermal imaging. ${ }^{6,7,12,13,14} \mathrm{To}$ what extent cryotherapy alters temperature of deep muscle tissue is of clinical importance and interest in the literature. ${ }^{1,15}$ Although previously argued that $\mathrm{T}_{s k}$ was a weak predictor of deeper tissue temperatures, ${ }_{1}^{1}$ subsequent research presented a significant quadratic relationship between $\mathrm{T}_{s k}$ and intramuscular temperature $\left(\mathrm{T}_{i m}\right) .^{3}$ This developed knowledge regarding the physiological effects of cooling in areas other than $\mathrm{T}_{\text {sk }}$ relevant to cryotherapy applications in sport. Thermodynamic properties of cryotherapy modalities are reported with differences noted between the cooling abilities of crushed ice, wetted ice, and gel packs. ${ }^{16}$ Further differences between frozen peas, crushed ice, gel packs, and ice-water immersion are also reported. ${ }^{2}$ In addition, it has been suggested that pre-application modality temperatures do not influence the effectiveness of a cryotherapeutic modality in terms of $\mathrm{T}_{\text {sk }}$ reduction, however discerning the type of modality to be used with consideration of other variables in clinical decision-making is necessary. ${ }^{2}$

The magnitude of soft tissue temperature change caused by physiological response to cryotherapy is subject to the interaction of four factors. ${ }^{17}$ These include consideration of Fourier's Law, length of cooling exposure, heat capacity of the cooled area relating to thermal conductivity, and the thermodynamic properties of the cooling modality. ${ }^{17}$ Adipose tissue levels affect the clinical effectiveness of cooling ${ }^{8}$ and therefore influence the decisions regarding cooling dose or choice of modality. Although magnitude and depth of cooling into $\mathrm{T}_{\text {im }}$ have been investigated, ${ }^{1,2,3,8}$ research has not explored this in any sport specific context. Early literature examining temperature change in deep tissue inversely relates to skinfold levels (as a measure of body fat) and limb circumference. ${ }^{18}$ However, several studies have failed to report on the heterogeneities of participant characteristics or physical properties of the cryotherapy modalities. A clinically important relationship between adipose thickness and required cooling time exists, suggesting that an adjustment to application duration of crushed ice is required to produce similar $\mathrm{T}_{i m}$ temperature changes, dependent on skin fold measurements. ${ }^{19}$ Lipocytes, present in adipose tissue and low in diffusivity and conductivity, conserve heat from underlying tissues therefore acting as an insulating layer. ${ }^{3}$ Earlier authors have reported similar observations when exploring relationships between adipose, subcutaneous tissue, and depth of cooling achieved. ${ }^{18,20}$ Although, to the authors' knowledge no profiling of $\mathrm{T}_{s k}$ data relating physiological responses to cooling within specific sporting positions in rugby union is available. Examining these factors may be useful in the design of appropriate cooling protocols in sporting contexts.

In the sport of rugby union, characteristics vary between playing position ${ }^{21}$ demonstrating a diverse range of anthropometric attributes. ${ }^{22}$ This multiplicity of bodily appearance across players supports the both the demands of each playing position and performance differences seen across the sport. ${ }^{22}$ Homogeneity of form and performance attributes is less common in rugby union, presenting it as an 'atypical' sport in comparison to other team sports, ${ }^{22}$ with playing positions commonly referred to as 'forwards' or 'backs'. ${ }^{23}$ These positional differences are centered around game demands. ${ }^{24,25}$ Subsequently, some authors have presented further differences in movement characteristics in these groups. ${ }^{26,27}$ Consistent with previous research on physical traits of rugby 
union players the current study provides comparison regarding several anthropometric parameters and characteristics, such as body mass, which has been found to be greater in forwards compared to backs. ${ }^{28}$ This larger size for forwards corresponds to the consideration of force-generation required in the scrum, ${ }^{29}$ with extra mass traditionally consisting of adipose tissue rather than lean tissue. ${ }^{22}$ However, in recent years, this has changed with forwards presenting with lower levels of body fat to enable increased mobility generally required in elite level participation. ${ }^{30}$ Literature suggests that differences in body fat percentage between levels of play also exist, with non-elite populations presenting with higher levels of adipose tissue. ${ }^{22}$ In consideration of the diverse physical traits present in rugby union players and the known interference between adipose tissue and the ability to cool deeper tissues, further evidence is required to determine optimal application of cryotherapy in this population. Additionally, comparison of cooling between commonly applied cryotherapeutic modalities in sport is insufficiently described. Therefore, the purpose of the current study was to investigate differences in the cooling ability of three different cryotherapy modalities in a rugby union population in an attempt to describe optimum cooling protocols for the anterior thigh.

\section{METHODS}

\section{Design}

Within subjects randomized crossover trial.

\section{Participants}

Inclusion criteria required participants to be male, take part in team, competitive rugby union across University, RFU National One or Two English league level. Due to consideration of gender differences in response to cooling an all-male population was sought. ${ }^{31}$ All participants were required to Exclusion criteria included any contraindications to cryother$a_{p y}{ }^{2}$ previous knee joint surgery, any lower limb injuries in the prior six months, or referred pain either to or from the knee. All participants provided written consent to take part in the study and completed a Physical Activity Readiness Questionnaire (PAR-Q) prior to participation in the study. The study was conducted according to the Declaration of Helsinki and approved by the host university ethics committee.

\section{Procedures}

Data were collected in a rugby union clinical setting with ambient room temperature collected to note any noteworthy fluctuations in room temperature during testing; mean ambient room temperature was recorded at $21.5 \pm 1.2^{\circ} \mathrm{C}$. A 15 -minute acclimatization period-allowed participant temperature equilibrium to take place prior to baseline data collection during which the collection of participants' height, weight, dominant leg, age, and thigh circumference was completed. Skinfold measures using Harpenden Skinfold Callipers (model HSB-BI; Baty International, Burgess Hill, West Sussex, UK), were used to estimate the percentage body fat based on the sum of skin fold thickness for adipose tissue measurements taken from the following sites: thigh, abdomen, medial calf, triceps, biceps, iliac crest, supraspinatus and subscapularis. ${ }^{32}$ Quantification of anthropometric assessment commonly considers body fat percentage via the collection of skinfold measurement. Although errors in precision of skin fold testing occur, research suggests that it is common practice in elite groups of athletes across sports. $^{22}$

Participants were randomly assigned following acclimatization, each participant to one method of cryotherapy intervention (Randomisation.com). Prior to application of cryotherapy, baseline measurements were taken, via three $\mathrm{T}_{s k}$ images of the anterior thigh, using a thermal imaging camera (ThermoVision A40M, Flir Systems, Danderyd, Sweden) and the mean of these measurements was used for data analysis. The anterior thigh location was chosen as the area to investigate because it is considered as a common site for contact injury, such as a contusion. In order to standardize an area of interest relevant to each participant in consideration of individual size differences and characteristics, wooden markers were applied to the non-dominant anterior thigh that defined a region of interest (ROI). This ROI was formulated by measuring the circumference of the thigh, 50\% between anatomical points of the greater trochanter of the femur and lateral joint line of the knee; circumference of the thigh served as the horizontal axis of the ROI. ${ }^{33}$ The vertical axis of the ROI measured from the anterior superior iliac spine to the superior pole of the patella. ${ }^{34}$ Where horizontal 
and vertical axes merge, central ROI was determined. ${ }^{33,34}$ Superior to inferior borders of the ROI represented as one third of the ASIS to superior pole of patella measurement. Medial to lateral borders of the ROI represented as $25 \%$ of the circumference of the thigh (Figure 2). ${ }^{33,34}$ Once the ROI was determined, skin was marked with a washable pen and wooden markers applied to the determined locations (Figure 2), and pre-intervention images were taken.

Emissivity of the thermal imaging camera was set at 0.97-0.98 following standard medical protocols. Thermacam Researcher Pro 2.8 software was used to analyse skin temperature images. The thermal imaging camera positioned over a plinth, facing inferiorly to allow participants to remain in a semirecumbent supine position during application of the intervention and for additional measurements of the $\mathrm{T}_{\text {sk }}$ over the anterior thigh. The camera was mounted on a tripod arranged at a distance between the camera and participant ranging from 1.5-2.m dependent on limb size for image focus.

Participants were exposed to three different cryotherapy modalities, Crushed Ice (CI), Thigh CryoCuff( (DJO Global, Surrey, UK) (CC), and Wetted Ice (WI) in a random order. Each modality was applied for 20 minutes. ${ }^{4,19}$ CI consisted of $800 \mathrm{~g}$ of crushed ice in a clear 22x40cm 1-mil polyethene bag with excess air removed secured over the anterior thigh with plastic wrap; WI consisted of $500 \mathrm{~g}$ of crushed ice combined with $500 \mathrm{ml}$ of water in a $22 x 40 \mathrm{~cm} 1$-mil polyethene bag secured with plastic wrap; CC prepared using a thigh wrap attachment and the standard Cryo/Cuff ${ }^{\circledR}$ tub filled, half water and half crushed ice to the advised limit. A standard cling wrap held in place the CI and WI during testing. Each exposure was separated by at least seven days, ${ }^{12}$ according to the within subjects randomized crossover design study. Subjects were asked to refrain from ingestion of caffeine, food or alcohol and energetic exercise and for at least two hours prior to the application of the icing modalities. ${ }^{34}$

Each participant recorded thermal sensation and comfort ratings ${ }^{35}$ pre- and post-intervention for each condition (CI, CC and WI). Thermal sensation $^{35}$ was measured by asking the question: 'How are you feeling now?' Participants responded by grading the sensation of temperature relevant to their anterior thigh, on a standardized scale from -4 to $4(-4=$ very cold,$-3=$ cold,$-2=$ cool, $-1=$ slightly cool, $0=$ neutral,$+1=$ slightly warm, $+2=$ slightly hot,$+3=$ hot,$+4=$ very hot). Thermal comfort ${ }^{12,36}$ was determined by asking participants the question: 'Do you find this...?'. Participants answered using a five-point scale, where $0=$ comfortable, $1=$ slightly comfortable, 2 = uncomfortable, 3 =very uncomfortable, $4=$ extremely uncomfortable. After completion of the 20-minute cryotherapy exposure and removal of the modality, five thermal images of the anterior thigh were taken and used for data analysis.

\section{STATISTICAL ANALYSIS}

Data were analyzed using a repeated measures model (SPSS Version 24, SPSS Inc. Chicago, IL), using data pre-exposure as a covariate when comparing between all three applications of cryotherapy, applying least significant pairwise comparisons. The distribution of data about the mean were assessed and found to be suitable for parametric testing for $\mathrm{T}_{\text {sk. }}$. Non-parametric Friedman tests were used for comparison of thermal comfort and sensation data to explore differences between applications of cryotherapy. ${ }^{12}$

\section{RESULTS}

Twenty-one rugby union players $(20 \pm 2.9$ years, body mass $96.2 \pm 16.7 \mathrm{Kg}$, height $179.9 \pm 7.1 \mathrm{~cm}$, BMI $29.7 \pm 2.6 \mathrm{~kg} / \mathrm{m} 2$ and thigh circumference $62.5 \pm 7.1 \mathrm{~cm})$ volunteered to take part in the study (Figure 1).

\section{SKIN SURFACE TEMPERATURE $\left(\mathrm{T}_{S K}\right)$}

\section{$\mathbf{T}_{\text {sk }}$ Whole Group}

When comparing whole group baseline $\mathrm{T}_{\mathrm{sk}}$ to immediately post removal $\mathrm{T}_{s k}$ statistically significant reductions in $\mathrm{T}_{s k}$ occurred for all three applications, CI $(p=0.000)$, CC $(p=0.000)$ and WI $(p=0.000)$. A statistically significant difference was observed in $\mathrm{T}_{\text {sk }}$ when comparing post application CI to CC $(p=0.000)$, with CI producing significantly cooler $\mathrm{T}_{s k}$ than CC (Table 1). Additionally, a statistically significant difference in $\mathrm{T}_{s k}$ was noted when comparing post WI to CC $(p=0.01)$ with WI producing significantly cooler $\mathrm{T}_{s k}$ than CC (Table 1). No significant differences in 


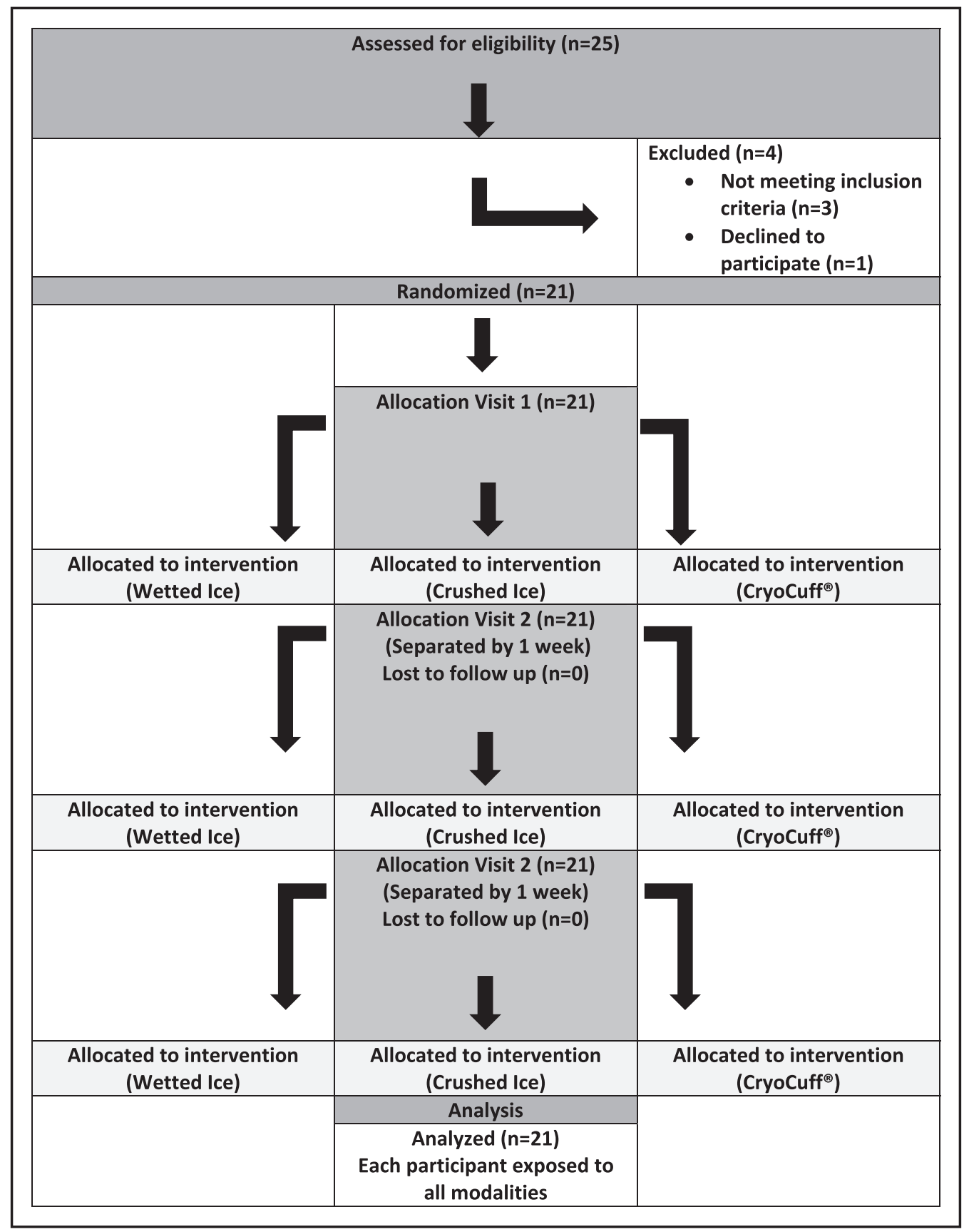

Figure 1. CONSORT diagram, demonstrating flow of participant recruitment, allocation and analysis.

$\mathrm{T}_{s k}$ were demonstrated when comparing between CI and WI $\mathrm{T}_{\text {sk }}$ post intervention $(p=0.141)$.

\section{$\mathbf{T}_{\text {sk }}$ Comparisons between Forwards and Backs}

Statistically significant differences in $\mathrm{T}_{s k}$ were noted when comparing playing positions, with significantly greater reductions in $\mathrm{T}_{s k}$ immediately post-intervention in the 'forwards' position group $(p=<0.05)$ compared to the backs' position group for, all three modalities $(p=<0.05)$, (Table 1$)$.

\section{Thermal Comfort and Thermal Sensation}

\section{Whole Group}

There was a significant decrease in reported thermal comfort post intervention when compared to pre-intervention measures for CI $(p=0.014)$, WI $(p=0.014)$ and CC $(p=0.025)$. No significant differences were noted when comparing between modalities for thermal comfort $(p=0.755)$. There were significant decreases in reported thermal sensation post-intervention when compared to 
pre-intervention measures for CI $(p=0.001)$, WI $(p=0.000)$ and CC $(p=0.000)$. No significant differences were noted when comparing between modalities for thermal sensation $(p=0.225)$ (Table 1$)$.

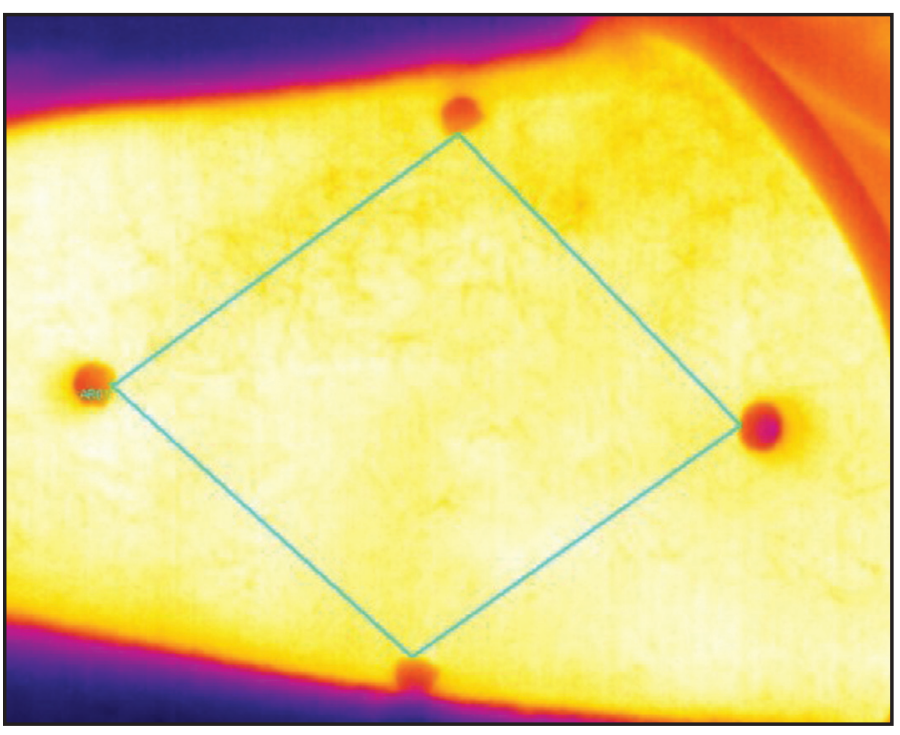

Figure 2. Region of Interest (ROI) over the anterior thigh of the non-dominant limb determined by percentage circumference and anatomical location points for each participant.

\section{Thermal Comfort and Thermal Sensation for Forwards and Backs}

With data collapsed into positional groupings of forwards and backs, a statistically significant decrease in thermal comfort post-intervention when compared to pre-intervention measures was displayed for WI in the forwards group ( $p=\leq 0.05)$. No significant change in thermal comfort was displayed for CI or CC in the forwards group ( $p=>0.05$ ) (Table 1). A statistically significant decrease in thermal comfort post-intervention when compared to preintervention measures was displayed for both WI $(p=0.014)$ and CI $(p=0.014)$ in the backs group. No significant change in thermal comfort was displayed for CC in the backs group ( $p=>0.05$ ) (Table 1). A significant decrease in thermal sensation was observed post intervention when compared to preintervention for CI, WI, and CC in both forwards and backs groups $(p=\leq 0.05)$ (Table 1$)$. No statistically significant differences were observed in thermal comfort or thermal sensation in both forwards and backs groups, when comparing between any modality $(p=\geq 0.05)$.

Table 1. Skin Temperature $\left(T_{\text {sk }}\right)$, self-reported scale for thermal comfort and thermal sensation data for whole group, and rugby union positional groups of forwards and backs.

\begin{tabular}{|c|c|c|c|c|c|c|c|c|c|}
\hline $\begin{array}{l}\text { Participant } \\
\text { (Groups) }\end{array}$ & $\begin{array}{l}\text { Body Fat } \\
\%\end{array}$ & $\begin{array}{l}\text { Average } \\
\text { Thigh } \\
\text { Circumference } \\
\text { (cm) }\end{array}$ & $\begin{array}{l}\text { Time Point / } \\
\text { Modality }\end{array}$ & $\mathrm{T}_{s k}\left({ }^{\circ} \mathrm{C}\right)$ & \begin{tabular}{|l|}
$\mathrm{T}_{s k}$ \\
(p-value \\
immediately post \\
compared to \\
baseline) \\
\end{tabular} & $\begin{array}{l}\text { Thermal } \\
\text { Comfort } \\
\text { (Average } \\
\text { Score) }\end{array}$ & $\begin{array}{l}\text { Thermal Comfort } \\
\text { (p-value } \\
\text { immediately post } \\
\text { compared to } \\
\text { baseline) } \\
\end{array}$ & $\begin{array}{l}\text { Thermal } \\
\text { Sensation } \\
\text { (Average } \\
\text { Score) }\end{array}$ & $\begin{array}{l}\text { Thermal Sensation } \\
\text { (p-value } \\
\text { immediately post } \\
\text { compared to } \\
\text { baseline) } \\
\end{array}$ \\
\hline \multirow[t]{5}{*}{$\begin{array}{l}\text { Whole group } \\
\mathbf{n}=21\end{array}$} & \multirow[t]{5}{*}{$21.0 \pm 6.7$} & \multirow[t]{5}{*}{$61.5 \pm 5.2 \mathrm{~cm}$} & \begin{tabular}{|l|} 
PRE \\
INTERVENTION
\end{tabular} & $29.9 \pm 1.1^{\circ} \mathrm{C}$ & - & 0 & - & 0 & - \\
\hline & & & IP (CI) & $13.0 \pm 2.1^{\circ} \mathrm{C}$ & $\mathrm{p}=0.000^{*}$ & 1 & $\mathrm{p}=0.014^{*}$ & -2 & $\mathrm{p}=0.000^{*}$ \\
\hline & & & $\mathrm{IP}(\mathrm{CC})$ & $15.8 \pm 1.4^{\circ} \mathrm{C}$ & $\mathrm{p}=0.000^{*}$ & 0 & $\mathrm{p}=0.025^{*}$ & 1 & $\mathrm{p}=0.001^{*}$ \\
\hline & & & IP (WI) & $12.0 \pm 3.0^{\circ} \mathrm{C}$ & $\mathrm{p}=0.000^{*}$ & 1 & $\mathrm{p}=0.014^{*}$ & -3 & $\mathrm{p}=0.000^{*}$ \\
\hline & & & & - & - & - & - & - & - \\
\hline \multirow[t]{5}{*}{$\begin{array}{l}\text { Forwards } \\
n=12\end{array}$} & \multirow[t]{5}{*}{$23.3 \pm 6.3$} & \multirow[t]{5}{*}{$64.3 \pm 7.9 \mathrm{~cm}$} & \begin{tabular}{|l|} 
PRE \\
INTERVENTION
\end{tabular} & $29.2 \pm 1.0^{\circ} \mathrm{C}$ & - & 0 & - & 0 & - \\
\hline & & & IP (CI) & $12.6 \pm 2.0^{\circ} \mathrm{C}$ & $\mathrm{p}=0.000^{*}$ & 0 & $\mathrm{p}=>0.05$ & -2 & $\mathrm{p}=0.000^{*}$ \\
\hline & & & IP (CC) & $15.3 \pm 1.3^{\circ} \mathrm{C}$ & $\mathrm{p}=0.000^{*}$ & 0 & $\mathrm{p}=>0.05$ & 1 & $\mathrm{p}=0.001^{*}$ \\
\hline & & & IP (WI) & $10.9 \pm 2.6^{\circ} \mathrm{C}$ & $\mathrm{p}=0.000^{*}$ & 1 & $\mathrm{p}=0.014^{*}$ & -3 & $\mathrm{p}=0.000^{*}$ \\
\hline & & & & - & - & - & - & - & - \\
\hline \multirow[t]{5}{*}{$\begin{array}{l}\text { Backs } \\
\mathrm{n}=9\end{array}$} & \multirow[t]{5}{*}{$15.4 \pm 4.1$} & \multirow[t]{5}{*}{$57.8 \pm 2.4 \mathrm{~cm}$} & \begin{tabular}{|l|} 
PRE \\
INTERVENTION
\end{tabular} & $30.7 \pm 0.5^{\circ} \mathrm{C}$ & - & 0 & - & 0 & - \\
\hline & & & IP (CI) & $14.5 \pm 1.7^{\circ} \mathrm{C}$ & $\mathrm{p}=0.000^{*}$ & 1 & $\mathrm{p}=0.014^{*}$ & -2 & $\mathrm{p}=0.000^{*}$ \\
\hline & & & $\mathrm{IP}(\mathrm{CC})$ & $17.0 \pm 0.8^{\circ} \mathrm{C}$ & $\mathrm{p}=0.000^{*}$ & 0 & $\mathrm{p}=>0.05$ & 1 & $\mathrm{p}=0.001^{*}$ \\
\hline & & & IP (WI) & $15.4 \pm 1.6^{\circ} \mathrm{C}$ & $\mathrm{p}=0.000^{*}$ & 1 & $\mathrm{p}=0.014^{*}$ & -2 & $\mathrm{p}=0.000^{*}$ \\
\hline & & & & - & - & - & - & - & - \\
\hline $\begin{array}{l}\text { Tsk }=\text { Skin su } \\
\text { represents a p } \\
\text { scale grades } \mathrm{t} \\
*=\text { Significan }\end{array}$ & emper & $\begin{array}{l}\text { IP = Immedi } \\
\text { of 'comfortab } \\
\text { aperature from } \\
\text { ed compared }\end{array}$ & cooling. $\mathrm{CI}=$ & dice. & CryoCuff $^{\mathbb{B}}$. & ice. $\mathrm{T}$ & 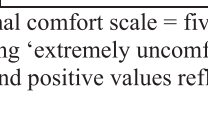 & int scale & $\begin{array}{l}0-4 \text {, a zero response } \\
\text { nsation scale }=\text { the }\end{array}$ \\
\hline
\end{tabular}




\section{DISCUSSION}

Despite the common application techniques of cryotherapeutic modalities and their use in sports injury management, consensus for optimum protocols are inconsistent across literature..$^{1,2,3,4}$ The present study investigated a comparison of three cooling modalities frequently applied in the sporting context for pitch-side management of soft tissue injury in a rugby union population. CI, CC and WI were applied on separate occasions over the anterior thigh in a rugby union population, representing all playing positions, sub-grouped into 'forwards' and 'backs'. Results suggest differences in the ability to reduce $\mathrm{T}_{s k}$ to within the desired therapeutic range of cooling occurs between cryotherapeutic modalities, in line with similar investigations. ${ }^{2}$ Comparison between modalities for whole group data, reported differences in $\mathrm{T}_{s k}$, with WI displaying the greatest reduction of $\mathrm{T}_{\text {sk }}\left(12.0 \pm 3.0^{\circ} \mathrm{C}\right)$ and $\mathrm{CC}$ offering the least reduction in $\mathrm{T}_{s k}\left(15.8 \pm 1.4^{\circ} \mathrm{C}\right)$ recorded immediately post removal. Evaluation of $\mathrm{T}_{s k}$ between the two positional groups; 'forwards' compared to 'backs', demonstrated significant differences in $\mathrm{T}_{\text {sk }}$ between positional groups and between types of applications $(p=>0.05)$. These findings have implications on optimal cooling protocol development and application choice when applied to a rugby union population.

When comparing whole group $\mathrm{T}_{s k}$ data to baseline, most modalities (CI, WI) demonstrated the ability to cool $\mathrm{T}_{\text {sk }}$ to within the desired therapeutic range of $10-15^{\circ} \mathrm{C}$ (Table 1 ). ${ }^{37,38} \mathrm{~T}_{\text {sk }}$ findings suggest these modalities may be capable of initiating positive physiological responses within deeper tissues, such as intramuscular cooling determined through observation of $\mathrm{T}_{\text {sk }}$ in consideration of the proposed quadratic relationship reported in earlier literature. ${ }^{3}$ Although, aside from $\mathrm{T}_{\text {sk }}$ measures, other physiological responses were not quantified in this study, therefore, the suggested responses can only be assumed to occur based on previous literature. ${ }^{3}$

Observations of $\mathrm{T}_{s k}$ displayed different responses between modalities supporting previous literature ${ }^{2,5,17,39}$ with WI achieving the coldest average $\mathrm{T}_{\text {sk }}$ in whole group data $\left(12.0 \pm 3.0^{\circ} \mathrm{C}\right)$ compared to CC and CI. Interestingly, when comparing positional groups, WI application achieved the coolest $\mathrm{T}_{\text {sk }}$ in the 'forwards' group $\left(10.9 \pm 2.6^{\circ} \mathrm{C}\right)$, but not in the 'backs' group $\left(15.4 \pm 1.6^{\circ} \mathrm{C}\right)$ Forwards demonstrated a larger thigh circumference compared to backs (forwards $=64.3 \pm 7.9 \mathrm{~cm}$; backs $=57.8 \pm 2.4 \mathrm{~cm})$, which was accompanied by a higher body fat percentage (forwards $=23.3 \pm 6.3 \mathrm{~cm} ;$ backs $=15.4 \pm 4.1 \mathrm{~cm}$ ). Contrary to what was expected, forwards displayed lower $\mathrm{T}_{s k}$ across all modalities compared to the backs. Indicating, that an increased adipose tissue has an effect on superficial $\mathrm{T}_{s k}$ responses. Consideration of the process of conduction and the insulating dynamics that adipose tissue presents however provides an explanation as to why participants in the 'forwards' group illustrated lower $\mathrm{T}_{\text {sk. }}$. It could be postulated that heat was more efficiently extracted in the group with lower adipose tissue (backs). Consequently, at the point of application removal (20 minutes) $\mathrm{T}_{s k}$ may have already begun to demonstrate 'rewarming' of the superficial tissues in this group (backs) due to efficient heat extraction from deeper tissues. This was represented by higher $\mathrm{T}_{s k}$ compared to the forwards group at the same time point. It was considered that the forwards groups displayed cooler $\mathrm{T}_{s k}$ because the higher levels of adipose tissue reduces efficient extraction of heat at the same capacity when compared to those with lower levels of adipose tissue. This would agree with earlier assumptions suggesting a dilution of net loss of heat lost to the cold modality ${ }^{16}$. With that in mind we assume that deeper tissues that are also of greater distance from the cooling modality were negatively affected in terms of intramuscular temperature reduction by the levels of insulating tissues. Although this principle is not unknown by any means, the discussion highlights that on the surface although data appears to show more efficient cooling of $\mathrm{T}_{s k}$ in the group with higher levels of adipose tissue (forwards) compared to the group with lower adipose tissue (backs), due to cooler $\mathrm{T}_{s k}$ reported. We presume on this basis that deeper physiological responses were likely affected differently amongst positional groups in the current study. Findings represent specific sporting populations not previously investigated. Sports physical therapists should consider these implications for optimal cryotherapy protocol development within specific population groups that present with distinct physical characteristic differences in relation to adipose tissue. 
Comparison of cooling distribution between modalities as examined visually via thermal imaging may support the post-intervention $\mathrm{T}_{s k}$ values (Table 1 ), with CC demonstrating an uneven distribution in cooling of the anterior aspect of the thigh, compared to WI or CI. This appeared to be consistent among participants, regardless of position. This pattern of cooling may be due to the potential uneven contact between the CC modality and skin surface, potentially facilitated by the compression of the device around the limb when expansion of the wrap commences following the introduction of fluid flow into the cuff. Although considered perhaps as a negative when attempting to achieve a significant or consistent cooling response over skin surface, the CC modality is advantageous in respect to the size of the area covered and the ability to provide circumferential pressure around the entire thigh, compared to the smaller targeted anterior limb region covered by the applications of CI and WI (contained in polythene bags secured by plastic wrap). Optimal compression protocols are unknown and further investigation of contemporary cryo-compressive products are warranted in terms of magnitudes of cooling with the adjunct of compression. Furthermore, investigations that consider targeted treatment vs circumferential over not only muscle but joint structures may be beneficial for future development of optimal cryotherapy protocols in sport.

The differences in $\mathrm{T}_{s k}$ between modalities reported in the current study for whole group data (Table 1) may be explained by phase change capability. Phase change in terms of thermodynamics is explained as the efficacy of cooling modalities to absorb heat. Modes of cooling differ in respect to their phase change ability and consequently demonstrated by the magnitude of $\mathrm{T}_{s k}$ reduction achieved post application. ${ }^{16,17}$ Findings in the current study are in line with previous literature that suggest CI is particularly effective in latent heat transfer. ${ }^{2}$ Our results demonstrate lower $\mathrm{T}_{s k}$ temperatures for CI and WI compared to CC therefore suggesting greater phase change capability occurring in CI and WI modalities. Clearly the modality of water and crushed ice is efficient in reducing $\mathrm{T}_{s k^{\prime}}$ therefore an explanation as to why CC did not achieve a therapeutic cooling range in whole group data may be explained simply as the poor conductivity of the interface material in contact with the skin consequently affecting the ability of the ice-water to extract heat. Again this may be mitigated by longer application dose, of which needs further investigation to develop optimal contemporary cryotherapeutic modality applications in sport. A dose of 30-minutes CI over the quadriceps in a similar fashion reported by Merrick et al. ${ }^{16}$ achieved slightly cooler reductions in $\mathrm{T}_{\text {sk }}$ suggesting longer applications influence resultant $\mathrm{T}_{s k}$. This in turn has an effect on deeper intramuscular temperature. ${ }^{3,16}$ In summary the results of the current study suggest that some modalities may be more appropriate for the acute management of sports injuries than others due to their phase change ability.

Thermal comfort and sensation outcomes demonstrated predictable reports in response to cooling and cold temperatures, that being self-reported reductions across both scores (Table 1) for the modalities with lower $\mathrm{T}_{\text {sk }}$ reductions (WI and CI). The modality with the lesser reduction in $\mathrm{T}_{s k}(\mathrm{CC})$ demonstrated no change in comfort scores despite reductions in $\mathrm{T}_{\text {sk }}$ occurring, however interestingly sensation was reported to increase from 0 (neutral) to 1 (slightly warm), evident across both whole group and sub group data (Table 1). When observing different responses in thermal comfort between forwards and backs, WI achieved the same response being 'slightly uncomfortable'. The backs group also reported this for CI, but forwards reported no change in comfort for that particular modality. With regard to why one application is perceived as more of less comfortable, the findings may be due to the insulating effects of adipose tissue, notably as discussed earlier typically this is higher in forwards compared to backs, which may have influenced level of comfort interpreted. Considering thermal sensation scores, both CI and WI applications influenced a predicated reduction in sensation of temperature in both groups but interestingly the forwards group reported a lower feeling of cold for WI compared to the backs. It is unsure why this occurred but the findings correspond to the observed reductions in $\mathrm{T}_{\text {sk }}$ reported post removal, that being lower in the forwards than backs (Table 1). Subjective response to cooling in terms of comfort and sensation amongst different modalities has implications on optimal cryotherapy applications. 
Further investigation is required, and scores collected during application may be more beneficial rather than those collected once cooling had been removed.

It is difficult to compare current results with other published literature due to the number of variables, such as exposure times, thermodynamics of the modalities, population group, application protocol, and modality location in respect to $\mathrm{T}_{s k}$ responses. Although perhaps not directly comparable, the current results are likely relevant to contemporary discussions in the literature regarding optimum cooling protocols used both clinically and pitch-side for the management of injury. ${ }^{40}$ Additionally these results offer relevance regarding cryotherapy considerations within specific sporting populations where physical traits vary within a squad. Results support previous literature when considering the impact adipose tissue has on the effects of cooling modalities. ${ }^{1,8,19}$ Previous authors have encouraged clinicians to measure skinfold thickness in order to determine an appropriate cryotherapy duration. ${ }^{39}$ Adherence to identification, marking, and measurement of the defined site of skinfold testing is essential for accurate quantification of adipose tissue levels. ${ }^{41}$ Recommendations for treatment times based on target tissue depth suggest a minimum of 15 minutes of cryotherapy application to achieve $0-15 \mathrm{~mm}$ target tissue depth for cooling, ${ }^{40}$ but does not compare type of modality to best inform potential differences in application. Nor does the current evidence base investigate several modalities within specific sporting populations recognising the varying levels of adipose tissue related to positional characteristics. When collapsing the data into sub groups of forwards and backs, data indicates that adipose tissue levels representative of physical traits in the sport of rugby union affects $\mathrm{T}_{s k}$ response (Table 1 ). Findings are similar to previous literature and consider that adipose tissue levels dictate application dose. ${ }^{19}$ With this in mind however it is important to consider that all applications in the current study followed the same duration protocol of 20-minutes, with resultant differences in average $\mathrm{T}_{s k}$, post removal. Although dose exposure length (minutes) was not investigated in the current study, it is clear that adaptations in application protocols between modalities to achieve desired cooling should be considered. To achieve optimal treatment outcomes in response to cooling in rugby union populations, adaptations to dose therefore may be required as well as choice of modality, when applied to the anterior thigh. This agrees with previous suggestions in literature in relation to altering cooling dose in respect to adipose tissue levels, although presumptuous in the current study, as dose was standardised. ${ }^{19}$

The choice of cooling modality is an important part of clinical decision making in terms of treatment. ${ }^{2}$ Adaptations for individual cooling protocols not only regarding the choice of modality but also regarding the duration of exposure and dosage within safe limits is important. In a much larger sample, of multiple playing positions and elite levels, it may be advantageous to investigate positional subgroups further, such as the characteristics of forwards, such as props and locks compared to back row or hooker positions. Researchers have suggested that movement and game demands differ across playing positions, ${ }^{25}$ affecting the physical characteristics and possibly the interference for application of modalities. The development of a framework representing optimal cooling applications requires consideration of multiple variables behind the mechanisms of cryotherapy. This may support individual approaches to optimum cryotherapeutic protocols defined specifically by type of application, adipose tissue levels, ${ }^{19}$ the depth of target tissue to be cooled, ${ }^{40}$ and the circumference of thigh in rugby union populations for example. Comparison of cooling duration and compression adjuncts in future studies is of merit utilizing contemporary technological advances available in cooling modalities. In consideration of current practice for the management of muscular injury, further development of cooling protocols that investigate contemporary cooling devices should consider the impact on latent intramuscular changes post application, which may fluctuate between modalities as $\mathrm{T}_{s k}$ does.

\section{LIMITATIONS}

The generalizability of findings may be limited due to the voluntary participation of a healthy all-male population group of rugby union players. Due to differences in thermoregulation, adipose thickness 
and that all participants were non-injured, the use of these procedures cannot be assumed in other populations, such as those of different academy age, females, or injured populations that may respond differently. Specific consideration of further study of temperature changes that occur in injured populations is required.

\section{CONCLUSIONS}

Variability in approaches of cryotherapy application by Sports Physical Therapists, demonstrates a lack of consensus due to limited data to substantiate cryotherapy guidelines. ${ }^{40}$ The results of the current study concur with earlier research that suggests that differences in the cooling ability between cryotherapy applications exist. WI was able to produce the greatest decrease in $\mathrm{T}_{s k}$ corresponding with self-reported thermal comfort and sensation scores. The greatest implication of the current study supports recommendations to further the research in cryotherapy application to meet therapeutic goals through adaptation of protocols to each athlete. ${ }^{42}$ The significant variability in $\mathrm{T}_{s k}$ between cooling in the two positional groups affirms the importance of the individualization of local cooling protocols when considering physical traits and characteristics within a rugby union population. Future research may consider extending observation beyond the dichotomy of forwards/backs and to other sports in which variability in physical characteristics vary across a squad.

\section{REFERENCES}

1. Jutte LS, Merrick MA, Ingersol CD, Edwards JE. The relationship between intramuscular temperature, skin temperature, and adipose thickness during cryotherapy and rewarming. Archives Phys Med \& Rehab. 2001;82:845-850.

2. Kennet J, Hardaker N, Hobbs S, Selfe J. Cooling efficiency of 4 common cryotherapeutic agents. J Athl Train. 2007;42:343-348.

3. Hardaker N, Moss A, Richards J, Jarvis S, McEwan I, Selfe J. The relationship between skin surface temperatures measured via Non-contact Thermal Imaging and intra-muscular temperature of the rectus femoris muscle. Thermo Int. 2007;17: 45-50.

4. Bleakley C, Costello JT, Glasgow PD. Should athletes return to sport after applying ice. A systematic review of the effect of local cooling on functional performance. Sports Med. 2012;42:69-87.
5. Dykstra JH, Hill HM, Miller MG, Cheatham CC, Michael TJ, Baker RJ. Comparisons of cubed ice, crushed ice, and wetted ice on intramuscular and surface temperature changes. J Athl Train. 2009;44:136-141.

6. Selfe J, Hardaker N, Whittaker J, Hayes C. An investigation into the effect on skin surface temperature of three cryotherapy modalities. Thermol Int. 2009;19:121-126.

7. Costello J, McInerney C, Bleakley C, Selfe J, Donnelly A. The use of thermal imaging in assessing skin temperature following cryotherapy: A Review. J Thermal Bio. 2012;37:103-110.

8. Bleakley C, Glasgow P, Phillps N, Hanna L, Callaghan M, Davison G, Hopkins T and Delahunt E. Management of acute soft tissue injury using protection, rest, ice, compression and elevation recommendations from the Association of Chartered Physiotherapists in Sports and Exercise Medicine (ACPSM). Physio Sport, 2011;1:1-22.

9. Knight KL, Brucker JB, Stoneman PD, Rubley MD. Muscle injury management with cryotherapy. Athl Ther Today. 2000;5:26-30.

10. Algafly AA, George KP. The effect of cryotherapy on nerve conduction velocity, pain threshold, and pain tolerance. Br J Sports Med. 2007;41:365-369.

11. White G, Wells G. Cold-water immersion and other forms of cryotherapy: physiological changes potentially affecting recovery from high-intensity exercise. Extreme Physiol \& Med. 2013;2:2-11.

12. Selfe J, Alexander J, Costello JT, May K, Garratt N, Atkins S, et al. The effect of three different $\left(-135^{\circ} \mathrm{C}\right)$ whole body cryotherapy exposure durations on elite rugby league players. PLOS ONE. 2014;9:1-9.

13. Alexander J, Selfe J, Oliver B, Mee D, Carter A, Scott M, May K. An exploratory study into the effects of a 2 minute crushed ice application on knee joint position sense during a small knee bend. Phys Ther Sport. 2016;18:21-26.

14. Alexander J, Richards J, Attah O, Cheema S, Snook J, Wisdell C, et al. Delayed effects of a 20-min crushed ice application on knee joint position sense assessed by a functional task during a re-warming period. Gait Posture. 2018;62:173-178.

15. Enwemeka CS, Allen C, Avilla P, Bina J, Konrade J, Munns S. Soft tissue thermodynamics before, during and after cold pack therapy. Med Sci Sports Ex. 2002;34:45-50.

16. Merrick M, Jutte L, Smith M. Cold modalities with different thermo-dynamic properties produce different surface and intramuscular temperatures. J Athl Train. 2003;38:28-33. 
17. Von Nieda K, Michlovitz SL. Cryotherapy. In: Michlovitz SL (Ed). Thermal agents in rehabilitation 3rd Edition. F.A. Davies Company, Philadelphia. 1996.

18. Lowden BJ, Moore RJ. Determinants and nature of intramuscular temperature changes during cold therapy. Am J Phys Med. 1975;54:223-233.

19. Otte JW, Merrick MA, Ingersoll CD, Cordova ML. Subcutaneous adipose tissue thickness alters cooling time during cryotherapy. Arch Phys Med Rehab, 2002;83:1501-1505.

20. Zemke JE, Andersen JC, Guion K, McMillam J, Joyner B. Intramuscular Temperature Responses in the Human Leg to Two Forms of Cryotherapy: Ice Massage and Ice Bag. J Orthop Sport Phys Ther. 1998;27:301-307.

21. Cahill N, Lamb K, Worsfold P, Headey R, Murray S. The movement characteristic of English Premiership rugby union players. J Sports Sci. 2013;31:229-237.

22. Duthie GM, Pyne DB, Hooper S. The applied physiology and game analysis of rugby union. Sports Med. 2003;33:973-991.

23. Twist C, Worsfold P. (2014). Science of rugby. London: Routledge.

24. Duthie GM, Pyne DB, Hooper S. Time motion analysis of 2001 and 2002 Super 12 rugby. J Sports Sci. 2005;23:523-530.

25. Roberts SP, Trewartha G, Higgitt RJ, El-Abd J, Stokes KA. The physical demands of elite English rugby union. J Sports Sci. 2008;26:825-833.

26. Eaton C, George K. Position specific rehabilitation for rugby union players. Part 1: Empirical movement analysis data. Phys Ther Sport. 2006;7:22-29.

27. Deutsch MU, Kearney G, Rehrer N. Time-motion analysis of professional rugby union players during match play. J Sports Sci. 2007;25:461-472.

28. La Monica MB, Fukuda DH, Miramonti AA, Beyer KS, Hoffman MW, Boone CH, et al. Physical differences between forwards and backs in American collegiate rugby players. J Strength Cond Res. 2016;30:2382-2391.

29. Quarrie KL, Wilson BD. Force production in the rugby union scrum. J Sports Sci. 2000;18:237-246.

30. Olds T. The evolution of physique in male rugby union players in the twentieth century. J Sports Sci. 2001;19:253-262.
31. Cankar K, Finderle Z. Gender differences in cutaneous vascular and autonomic nervous response to local cooling. Clin Autonomic Res. 2003;13:214-220.

32. Duthie GM, Pyne DB, Hopkins WG, Livingstone S, Hooper SL. Anthropometry profiles of elite rugby players: quantifying changes in lean mass. $\mathrm{Br} \mathrm{J}$ Sports Med. 2006;40:202-207.

33. Doxey GE. The association of anthropometric measurements of thigh size and B-mode ultrasound scanning of muscle thickness. J Orthop Sports Phys Ther. 1987;8:462-468.

34. Janwantanakul P. The effect of quantity of ice and size of contact area on ice pack/skin interface temperature. Physiotherapy. 2009;95:120-125.

35. Cholewka A, Drzazga Z, Sieron A. Thermography study of skin response due to whole-body cryotherapy. Skin Res Technol. 2012;18:180-187.

36. International Organization for Standardization (ISO). Ergonomics of the thermal environment-assessment of the influence of the thermal environment using subjective judgement scales. International Organisation for Standardisation, Geneva, Switzerland, 2011.

37. Chesterton LS, Foster NE, Ross L. Skin temperature response to cryotherapy. Arch Phys Med Rehab. 2002;83:543-549.

38. Kanlayanaphotporn R, Janwantanakul P. Comparison of skin surface temperature during the application of various cryotherapy modalities. Arch Phys Med, Rehab. 2005;86:1411-1415.

39. Jutte LS, Hawkins JR, Miller KC, Long BC, Knight KL. Skinfold thickness at 8 common cryotherapy sites in various athletic populations. J Athl Train. 2012;47:170-177.

40. Hawkins JR, Miller KC. The importance of target tissue depth in cryotherapy application. J Athl Enhance. 2012;1:2.

41. Hume P, Marfell-Jones M. The importance of accurate site location for skinfold measurement. J Sports Sci. 2008;26:1333-1340.

42. Hawkins SW, Hawkins JR. Clinical applications of cryotherapy among sports physical therapists. Int $J$ Sports Phys Ther. 2016;11:141-148. 